\title{
Path-integral quantization of Galilean Fermi fields ${ }^{1}$
}

\author{
M. de Montigny ${ }^{a, b}$, F.C. Khanna ${ }^{a, c}$, F.M. Saradzhev ${ }^{a}$ \\ ${ }^{a}$ Theoretical Physics Institute, University of Alberta, \\ Edmonton, Alberta, Canada T6G 2J1 \\ ${ }^{b}$ Campus Saint-Jean, University of Alberta, \\ Edmonton, Alberta, Canada T6C 4G9 \\ ${ }^{c}$ TRIUMF, 4004, Westbrook Mall, \\ Vancouver, British Columbia, Canada V6T 2 A3
}

\begin{abstract}
The Galilei-covariant fermionic field theories are quantized by using the path-integral method and five-dimensional Lorentz-like covariant expressions of non-relativistic field equations. Firstly we review the five-dimensional approach to the Galilean Dirac equation, which leads to the Lévy-Leblond equations, and define the Galilean generating functional and Green's functions for positive- and negative-energy/mass solutions. Then, as an example of interactions, we consider the quartic self-interacting potential $\lambda(\bar{\Psi} \Psi)^{2}$, and we derive expressions for the 2- and 4-point Green's functions. Our results are compatible with those found in the literature on non-relativistic many-body systems. The extended manifold allows for compact expressions of the contributions in $(3+1)$ space-time. This is particularly apparent when we represent the results with diagrams in the extended $(4+1)$ manifold, since they usually encompass more diagrams in Galilean $(3+1)$ space-time.
\end{abstract}

\footnotetext{
${ }^{1}$ montigny@phys.ualberta.ca, khanna@phys.ualberta.ca, fsarajov@phys.ualberta.ca
} 


\section{Introduction}

Although its original successes lie in particle physics, quantum field theory has since then reached a much wider range of applications. Indeed, concepts such as perturbation methods and Feynman diagrams, renormalization group procedures, spontaneous symmetry breaking, etc. (both at zero and finite temperature) have been interchangeably utilized by physicists working in particle physics as well as in condensed matter physics and statistical physics [1,2]. More modern field theories, such as conformal field theory, are applied in statistical physics, string theory, etc.

However, a fundamental difference between particle physics and condensed matter (or statistical) physics is that the latter involves the non-relativistic regime, whereas high-energy physics involves relativistic kinematics. In fact, field theoretical models typically are constructed by taking into account various symmetries, such as Poincaré space-time invariance. Recent achievements, such as Fermi condensates with ultracold potassium-40 atoms [3],suggest that analogous procedures should be devised for Galilean-invariant systems. Recent interest in the Galilean symmetry (particularly in the plane) is due to its applications to Hall efffect, anyons, Chern-Simons term, non-commutative geometry, etc [4].

This article is an extension to Fermi fields of a recent work where we have performed the path-integral quantisation of Galilean-invariant scalar fields [5]. It belongs to a series of papers whose general underlying program consists in using a formulation of Galilean covariance based on a relativistic framework in one higher dimension, which makes non-relativistic field theories similar to Lorentz-covariant theories [6]-[8]. In these articles, the extended manifold approach follows the lines of earlier investigations $[9,10]$. Similar approaches have been ubiquitous in physics $[11,12]$. Recently, it has been used in the study of fluid dynamics [13, 14].

The occurence of the $2+1$ Galilean group was observed [15] as the transverse motion to the direction of the infinite momentum frame, now better known as the light-cone frame, in a study of the perturbative behaviour in the limit of strong interaction processes. This has been suggested previously [16]. Later, this perspective was taken up in conjecturing an equivalence between eleven-dimensional M-theory and the $N=\infty$-limit of the supersymmetric matrix quantum mechanics which describes D0 branes [17] (These authors actually consider the super-Galilei group, which admits 32 real super-generators.)

Let us review the formalism briefly for our purposes. The algorithm henceforth consists in building action functionals by enforcing Lorentz covariance, as it is usually done with relativistic theories, except that Galilean kinematics is based on the so-

called Galilean five-vectors $\left(\mathbf{x}, x^{4}, x^{5}\right)$. These vectors transform under Galilean boosts as

$$
\begin{aligned}
\mathbf{x}^{\prime} & =\mathbf{x}-\beta x^{4}, \\
x^{4^{\prime}} & =x^{4},
\end{aligned}
$$




$$
x^{5^{\prime}}=x^{5}-\beta \cdot \mathbf{x}+\frac{1}{2}|\beta|^{2} x^{4}
$$

where $\beta \equiv \mathbf{v} / \bar{c}$, while $\mathbf{v}$ is the relative velocity between the two reference frames, and $\bar{c}$ is a parameter with the dimensions of velocity, which will be specified below.

Altogether the kinematical transformations, which also include rotations and translations, form a fifteen-dimensional Lie algebra. This may be seen as the Poincaré algebra in $(4+1)$ space-time. Eleven of these fifteen generators form the extended Galilei group, where the central-extension parameter (the non-relativistic mass) is inherited from the generator of $x^{5}$ translations. The transformation (1) for $x^{\prime 5}$ has occurred in various contexts [5]-[12]. In quantum mechanics, it is associated with the wave function's phase which enforces invariance of the Schrödinger equation under Galilean transformations. Furhermore, it leads to a superselection rule for mass conservation in Galilean covariant theories. Indeed, unlike the relativistic theories, new massive particles cannot be created in a Galilean framework. This would preclude Yukawa couplings except for massless particles with coupling to two massive particles. For massive particles, only the 4-particle coupling is allowed.

The invariant scalar product is defined as

$$
A \cdot B=A_{4} B_{5}+A_{5} B_{4}-\mathbf{A} \cdot \mathbf{B}
$$

with the Galilean metric:

$$
g_{\mu \nu}=\left(\begin{array}{ccc}
-\mathbf{1}_{3 \times 3} & 0 & 0 \\
0 & 0 & 1 \\
0 & 1 & 0
\end{array}\right)
$$

This suggests that the non-relativistic time is a light-cone parameter of the Lorentz invariant theory on a manifold containing one additional space-like dimension $[10,18]$. Once we have constructed a Galilean covariant action functional, an appropriate embedding of the Galilean space-time into $\mathcal{G}_{(4+1)}$ may be defined as

$$
(\mathbf{x}, t) \hookrightarrow x^{\mu}=\left(x^{1}, \cdots, x^{5}\right) \equiv\left(\mathbf{x}, \bar{c} t, \frac{s}{\bar{c}}\right)
$$

The five-momentum

$$
p_{\mu}=\mathrm{i} \partial_{\mu}=\left(\mathrm{i} \nabla, \frac{\mathrm{i} \partial_{t}}{\bar{c}}, \mathrm{i} \bar{c} \partial_{s}\right)=\left(\mathbf{p}, \frac{E}{\bar{c}}, m \bar{c}\right),
$$

where $p^{4}=p_{5}=m \bar{c}$ and $p^{5}=p_{4}=\frac{E}{\bar{c}}$, suggests that the additional coordinate $x^{5}=\frac{s}{\bar{c}}$ is canonically conjugated to $m \bar{c}$. From the relation $\partial_{s}=-\mathrm{i} m$, the phase factor of the wavefunction follows:

$$
\Psi(x) \equiv e^{-\mathrm{i} m s} \psi(\mathbf{x}, t)
$$


which projects the fields from $\mathcal{G}_{(4+1)}$ to $(3+1)$-dimensions. A different definition of dimensional reduction would lead to a Lorentz-covariant theory in $(3+1)$-dimensions $[10,11]$.

Note that it is also possible to define

$$
\Psi(x) \equiv e^{-\mathrm{i} m s} \psi_{+}(\mathbf{x}, t)+e^{+\mathrm{i} m s} \psi_{-}(\mathbf{x}, t)
$$

where $\psi_{ \pm}(\mathbf{x}, t)$ represent the positive- or negative-energy solutions, which makes evident the possibility of negative energy solutions [19]. This comes from the quadratic condition $\left(\partial_{s}\right)^{2}=-m^{2}$, and it is compatible with the embedding defined in Eq. (4) since additional terms with negative mass and negative energy can be included. Such a description is allowed by the symmetry $\left(\bar{c} t, \frac{s}{\bar{c}}\right) \rightarrow\left(-\bar{c} t,-\frac{s}{\bar{c}}\right)[20]$.

In $(4+1)$-dimensional Galilean theories, $p_{\mu} p^{\mu}=2 m E-\mathbf{p}^{2}$ is an invariant, and the dynamics of Galilean covariant fields must be consistent with it. Let us take

$$
p_{\mu} p^{\mu}=k^{2}
$$

where $k$ is a real constant that defines the invariant quantity. It leads to $2 m E-\mathbf{p}^{2}=$ $k^{2}$, which is analogous to $E^{2}-p^{2} c^{2}$, the invariant for Lorentz covariant fields that is equal to $m^{2} c^{4}$, thus defining $m$ as the invariant quantity. This implies the dispersion relation :

$$
E=\frac{1}{2 m}|\mathbf{p}|^{2}+\frac{1}{2 m} k^{2}
$$

Introducing a velocity parameter

$$
\bar{c}=\frac{k}{\sqrt{2} m},
$$

we cast the dispersion relation into the familiar form for non-relativistic energy with $E=\frac{1}{2 m}|\mathbf{p}|^{2}+m \bar{c}^{2}$. Note that this equation, as well as the invariant $2 m E-\mathbf{p}^{2}$, is invariant under the changes

$$
m \longrightarrow-m, \quad E \longrightarrow-E,
$$

so that in the $(4+1)$-manifold, one must reverse both $m$ and $E$, but not each one independently. Henceforth, we will have to ensure that this is satisfied when we split the positive- and negative-energy/mass solutions.

The constant $k$ is the Galilean analogue of the Lorentzian rest mass. Since $k$ can be absorbed within the energy $E$, its value is usually considered to be of no physical importance and taken to be zero. However, it may be possible to relate $k$ to the chemical potential [5]. For Galilean Fermi fields, the dispersion relation (5) implies that the negative energy solutions are characterized by negative masses.

The paper is organized as follows. In Section 2, we review the Lévy-Leblond equations by means of the extended-manifold Dirac equation, and the positive- and 
negative-energy/mass solutions, and we introduce the Galilean generating functional formalism. Appropriate embeddings associated with the virtual sources are defined. In Section 3, we establish the connection between the generating functional and the Green's functions for both positive- and negative energy/mass solutions. In Section 4, we apply this formalism to the self-interacting quartic potential. The 2- and 4-point functions are calculated. We distinguished between $(3+1)$ - and $(4+1)$-manifold diagrams, the latter containing, in general, more diagrams in the reduced $(3+1)$ space-time. Concluding remarks are in Section 5.

\section{Free Dirac field}

\subsection{Five-dimensional Dirac equation}

Let us consider a free Dirac field $\Psi(x)$ defined on the five-dimensional manifold $\mathcal{G}_{(4+1)}$ with Galilean metric, Eq. (2). Then a manifestly covariant Lagrangian for the Dirac field is given by

$$
\mathcal{L}_{0}=\bar{\Psi}(x)\left(\mathrm{i} \gamma^{\mu} \stackrel{\leftrightarrow}{\partial_{\mu}}-k\right) \Psi(x)
$$

where $a \overleftrightarrow{\partial b} \equiv \frac{1}{2}[a \partial b-(\partial a) b]$. Both the field and its adjoint are anticommuting. The matrices $\gamma^{\mu}$ in the extended space-time are four-dimensional and may be chosen as

$$
\gamma^{a}=\left(\begin{array}{cc}
\mathrm{i} \sigma^{a} & 0 \\
0 & -\mathrm{i} \sigma^{a}
\end{array}\right), \quad \gamma^{4}=\left(\begin{array}{cc}
0 & 0 \\
\sqrt{2} & 0
\end{array}\right), \quad \gamma^{5}=\left(\begin{array}{cc}
0 & \sqrt{2} \\
0 & 0
\end{array}\right),
$$

$\sigma^{a}, a=1,2,3$ denoting the $2 \times 2$ Pauli matrices. They obey the usual anticommutation relations :

$$
\left\{\gamma^{\mu}, \gamma^{\nu}\right\}=2 g^{\mu \nu}
$$

Let us apply the variational principle for the action integral with the free Lagrangian of Eq. (6),

$$
I[\Psi, \bar{\Psi}]=\int d^{5} x \mathcal{L}_{0}[\Psi, \bar{\Psi}]
$$

where the integral over $x^{5}$ is interpreted as $\int d x^{5} \rightarrow \lim _{l \rightarrow \infty} \frac{1}{l} \int_{-l / 2}^{l / 2} d x^{5}$, and $l$ is an arbitrary length. Then the Euler-Lagrange equations of motion for $\Psi(x)$ and its adjoint $\bar{\Psi}(x)$, respectively, are

$$
\left(\mathrm{i} \gamma^{\mu} \partial_{\mu}-k\right) \Psi(x)=0, \quad \bar{\Psi}(x)\left(\mathrm{i} \gamma^{\mu} \overleftarrow{\partial}_{\mu}+k\right)=0
$$

where $a \overleftarrow{\partial} b=(\partial a) b$. The adjoint field is defined as

$$
\bar{\Psi}(x)=\Psi^{\dagger}(x) \gamma^{0}
$$


where

$$
\gamma^{0}=\frac{1}{\sqrt{2}}\left(\gamma^{4}+\gamma^{5}\right)=\left(\begin{array}{ll}
0 & 1 \\
1 & 0
\end{array}\right) .
$$

Its reduction to $(3+1)$-dimensions is defined as

$$
\bar{\Psi}(x)=e^{\mathrm{i} m \bar{c} x^{5}} \bar{\psi}(\mathbf{x}, t)
$$

The first expression in Eq. (8), using Eqs. (3) and (4), reduces to

$$
\begin{aligned}
& ((\sigma \cdot \nabla)+k) \psi_{1}(\mathbf{x}, t)-\sqrt{2} m \bar{c} \psi_{2}(\mathbf{x}, t)=0 \\
& \sqrt{2} \frac{E}{\bar{c}} \psi_{1}(\mathbf{x}, t)+((\sigma \cdot \nabla)-k) \psi_{2}(\mathbf{x}, t)=0
\end{aligned}
$$

where

$$
\psi(\mathbf{x}, t)=\left(\begin{array}{c}
\psi_{1}(\mathbf{x}, t) \\
\psi_{2}(\mathbf{x}, t)
\end{array}\right) .
$$

with $\psi_{1}(x, t)$ and $\psi_{2}(x, t)$ being two-component spinors. The two equations in Eq. (10) are analogous to the Pauli equations in the relativistic case. These Galilean wave equations describe non-relativistic Fermi fields in $(3+1)$-dimensions. If $k=0$ then Eq. (10) coincides with the Lévy-Leblond equations [22]. The wave equations

for the adjoint Fermi fields have the same form and can be deduced from the second expression in Eq. (8) together with Eq. (9).

In analogy with the relativistic theory, we find that the Fourier components of the Galilean Dirac fields satisfy $\left(p_{\mu} p^{\mu}-k^{2}\right) \Psi(p)=0$ and $\left(p_{\mu} p^{\mu}-k^{2}\right) \bar{\Psi}(p)=0$, which reduce to the Schrödinger wave equations. Then each component of the $(3+1)$ dimensional non-relativistic Fermi fields obeys the Schrödinger equation :

$$
E \psi_{1,2}(p)=\left(\frac{\mathbf{p}^{2}}{2 m}+\frac{k^{2}}{2 m}\right) \psi_{1,2}(p) .
$$

Thus the Schrödinger equation may be obtained either by first reducing the Dirac equation to the Lévy-Leblond equations (10) with Eq. (4), or by first reducing Eq. (6) to the Lagrangian of the Schrödinger field.

\subsection{The positive- and negative-energy solutions and canoni- cal quantization}

The Lagrangian, Eq. (6), and the Dirac equations given by Eq. (8) are invariant with respect to unitary transformations

$$
\gamma^{\mu} \rightarrow S \gamma^{\mu} S^{-1}, \quad \Psi \rightarrow S \Psi
$$

where $S$ is a $4 \times 4$-matrix. To construct the positive- and negative-energy/mass solutions explicitly, it is convenient to use a representation in which $\gamma^{0}$ is diagonal. 
This representation can be obtained from the one used in the previous section by performing the unitary transformation above with the choice

$$
S=\frac{1}{\sqrt{2}}\left(\begin{array}{cc}
1 & 1 \\
1 & -1
\end{array}\right)
$$

In particular, $\gamma^{0}$ becomes

$$
\gamma^{0}=\left(\begin{array}{cc}
1 & 0 \\
0 & -1
\end{array}\right)
$$

It is important to point out that this form of $\gamma^{0}$ matrix does not imply chirality. In fact, there is no parity operator in five dimensions, hence no chirality. Only if we work in even dimensions, in this case six dimensions, can we find a parity operator, hence a chirality operator. Then the $\gamma$-matrices are 8 -dimensional. The details of this representation will appear elsewhere [23].

The matrices $\gamma^{\mu}$ take the form

$$
\gamma^{a}=\left(\begin{array}{cc}
0 & \mathrm{i} \sigma^{a} \\
\mathrm{i} \sigma^{a} & 0
\end{array}\right), \quad \gamma^{4}=\frac{1}{\sqrt{2}}\left(\begin{array}{cc}
1 & 1 \\
-1 & -1
\end{array}\right), \quad \gamma^{5}=\frac{1}{\sqrt{2}}\left(\begin{array}{cc}
1 & -1 \\
1 & -1
\end{array}\right)
$$

In what follows, we will use the representation defined by equations (11) and (12).

The plane-wave solutions for Eq. (8) are written in the usual form,

$$
\Psi^{(r)}(x)=\frac{1}{(2 \pi)^{5}} \int d^{5} p\left[u^{(r)}(p) e^{-i p x}+v^{(r)}(p) e^{i p x}\right]
$$

where $r=1,2$ and the positive- and negative-energy spinors $u^{(r)}(p)=u^{(r)}(\mathbf{p}, E, m)$, $v^{(r)}(p)=v^{(r)}(\mathbf{p}, E, m)$ obey the equations

$$
\left(\gamma^{\mu} p_{\mu}-k\right) u^{(r)}(p)=0, \quad\left(\gamma^{\mu} p_{\mu}+k\right) v^{(r)}(p)=0 .
$$

Taking the Dirac particle in the rest frame, $\mathbf{p}=0$, we find

$$
\gamma^{0} u^{(r)}(0)=u^{(r)}(0), \quad \gamma^{0} v^{(r)}(0)=-v^{(r)}(0),
$$

where

$$
\begin{aligned}
& u^{(r)}(0) \equiv u^{(r)}\left(0, E_{k}, m\right), \\
& v^{(r)}(0) \equiv v^{(r)}\left(0, E_{k}, m\right),
\end{aligned}
$$

and

$$
E_{k} \equiv \frac{k^{2}}{2 m}
$$

The representation with diagonal $\gamma^{0}$ is especially appropriate for describing particles at rest, the spinors $u^{(r)}(0), v^{(r)}(0)$ being eigenvectors of $\gamma^{0}$ with eigenvalues +1 and -1 , respectively. 
Let us define

$$
\xi^{(1)}(0)=\left(\begin{array}{l}
1 \\
0
\end{array}\right), \quad \xi^{(2)}(0)=\left(\begin{array}{l}
0 \\
1
\end{array}\right),
$$

which satisfy the relation

$$
\xi^{\dagger(r)}(0) \xi^{(s)}(0)=\delta_{r s} .
$$

We write the spinors $u^{(r)}(0), v^{(r)}(0)$ as

$$
u^{(r)}(0)=\left(\begin{array}{c}
\xi^{(r)}(0) \\
0
\end{array}\right), \quad v^{(r)}(0)=\left(\begin{array}{c}
0 \\
\xi^{(r)}(0)
\end{array}\right) .
$$

In a moving frame, the spinors $u^{(r)}(p), v^{(r)}(p)$ are expressed as

$$
u^{(r)}(p)=d_{u}\left(\gamma^{\mu} p_{\mu}+k\right) u^{(r)}(0), \quad v^{(r)}(p)=d_{v}\left(\gamma^{\mu} p_{\mu}-k\right) v^{(r)}(0) .
$$

These definitions are motivated by $p_{\mu} p^{\mu}=k^{2}$, so that $\left(\gamma^{\mu} p_{\mu}+k\right)\left(\gamma^{\mu} p_{\mu}-k\right)=p^{2}-k^{2}=$ 0 and Eq. (13) is satisfied.

The coefficients $d_{u}, d_{v}$ are computed from two conditions. Firstly, the right- and left-hand sides of Eq. (14) must coincide for $\mathbf{p}=0$, that is, $u^{(r)}(\mathbf{p}=0)=u^{(r)}(0)$ and $v^{(r)}(\mathbf{p}=0)=v^{(r)}(0)$. Secondly, the orthonormality conditions

$$
\bar{u}^{(r)}(p) u^{(s)}(p)=\delta_{r s}, \quad \bar{v}^{(r)}(p) v^{(s)}(p)=-\delta_{r s},
$$

which can be checked for the $\mathbf{p}=0$ case, must be valid for non-zero $\mathbf{p}$ as well. These two conditions determine $d_{u}$ and $d_{v}$ as

$$
d_{u}=-d_{v}=\frac{1}{2 k}\left(\frac{4 E_{k}}{E+3 E_{k}}\right)^{1 / 2}
$$

The general solution to the Galilean Dirac equations (8) may be expanded in terms of the plane wave solutions as

$$
\begin{aligned}
\Psi(x) & =\frac{1}{(2 \pi)^{3 / 2}} \sum_{r} \int d^{3} \mathbf{p}\left[a^{(r)}(\mathbf{p}) u^{(r)}(p) e^{-i p x}+b^{\dagger(r)}(\mathbf{p}) v^{(r)}(p) e^{i p x}\right] \\
\bar{\Psi}(x) & =\frac{1}{(2 \pi)^{3 / 2}} \sum_{r} \int d^{3} \mathbf{p}\left[a^{\dagger(r)}(\mathbf{p}) \bar{u}^{(r)}(p) e^{i p x}+b^{(r)}(\mathbf{p}) \bar{v}^{(r)}(p) e^{-i p x}\right]
\end{aligned}
$$

where $a^{(r)}(\mathbf{p})\left(a^{\dagger(r)}(\mathbf{p})\right)$ and $b^{(r)}(\mathbf{p})\left(b^{\dagger(r)}(\mathbf{p})\right)$ are destruction (creation) operators of particles and antiparticles, respectively. The fields are quantised by assuming that these operators obey the anticommutation relations:

$$
\left\{a^{(r)}(\mathbf{p}), a^{\dagger(s)}(\mathbf{q})\right\}=\left\{b^{(r)}(\mathbf{p}), b^{\dagger(s)}(\mathbf{q})\right\}=\delta_{r s} \delta(\mathbf{p}-\mathbf{q}) .
$$

All other anticommutation relations are zero. 
Using the non-relativistic "momentum-energy-mass" tensor

$$
T^{\mu \nu}=\frac{\partial \mathcal{L}}{\partial\left(\partial_{\mu} \Psi\right)} \partial^{\nu} \Psi+\partial^{\nu} \bar{\Psi} \frac{\partial \mathcal{L}}{\partial\left(\partial_{\mu} \bar{\Psi}\right)}-\mathcal{L} g^{\mu \nu},
$$

we define the five-momentum of the Galilean Dirac field as

$$
P_{\mu}=\int d^{3} x d x^{5} \sqrt{2} T_{5 \mu}=\frac{i}{\sqrt{2}} \int d^{3} x d x^{5}\left(\bar{\Psi} \gamma^{4} \partial_{\mu} \Psi-\partial_{\mu} \bar{\Psi} \gamma^{4} \Psi\right) .
$$

The charge operator is written as

$$
Q=\int d^{3} x d x^{5} \sqrt{2} \bar{\Psi} \gamma^{4} \Psi
$$

Substituting the expansions (15) and (16) into the expressions for $P_{\mu}$ and $Q$ and performing a normal ordering with respect to the vacuum state, we get

$$
a(\mathbf{k})|0\rangle=b(\mathbf{k})|0\rangle=0 \quad \text { for } \text { all } \mathbf{k} \text { and } m,
$$

giving, for the five-momentum and the charge operators,

$$
P^{\mu}=\sum_{r} \int d^{3} \mathbf{p} \cdot p^{\mu}\left[a^{\dagger(r)}(\mathbf{p}) a^{(r)}(\mathbf{p})+b^{\dagger(r)}(\mathbf{p}) b^{(r)}(\mathbf{p})\right],
$$

and

$$
Q=\sum_{r} \int d^{3} \mathbf{p} \cdot\left[a^{\dagger(r)}(\mathbf{p}) a^{(r)}(\mathbf{p})-b^{\dagger(r)}(\mathbf{p}) b^{(r)}(\mathbf{p})\right] .
$$

This corroborates the point, mentioned earlier, that $a^{\dagger}(\mathbf{p})$ and $a(\mathbf{p})$ are the creation and annihilation operators for particles of momentum $\mathbf{p}$, mass $m$ and charge +1 , whereas the operators $b^{\dagger}(\mathbf{p})$ and $b(\mathbf{p})$ correspond to antiparticles, which differ from the particles only by the sign of the charge, i.e. -1 .

\subsection{Galilean generating functional}

As in the usual path-integral formalism [24], the Galilean generating functional for the free field is given by the vacuum-to-vacuum transition amplitude with anticommuting virtual sources $J(x)$ and $\bar{J}(x)$ :

$$
Z_{0}[J, \bar{J}]=\int \mathcal{D} \bar{\Psi} \int \mathcal{D} \Psi \exp \left\{\mathrm{i} \int d^{5} x\left[\mathcal{L}_{0}[\Psi, \bar{\Psi}]+\bar{\Psi}(x) J(x)+\bar{J}(x) \Psi(x)\right]\right\},
$$

where $\int \mathcal{D} \bar{\Psi}$ and $\int \mathcal{D} \Psi$ denote the functional integrations over $\bar{\Psi}(x)$ and $\Psi(x)$, respectively. Here $J(x)$ and $\bar{J}(x)$ are anticommuting Grassmann virtual sources that we put equal to zero at the end.

Let us define a new field $\Psi^{\prime}$ :

$$
\Psi^{\prime}(x)=\Psi(x)-\Psi_{J}(x),
$$


where $\Psi_{J}(x)$ satisfies the inhomogeneous equation of motion :

$$
\left(\mathrm{i} \gamma^{\mu} \partial_{\mu}-k\right) \Psi_{J}(x)=-J(x) .
$$

Then we can complete the square within the exponential and rewrite the generating functional as

$$
\begin{aligned}
Z_{0}[J, \bar{J}]=\int \mathcal{D} \Psi^{\prime} \mathcal{D} \bar{\Psi}^{\prime} & \exp \left\{\mathrm{i} \int d^{5} x \mathcal{L}_{0}\left(\Psi^{\prime}, \bar{\Psi}^{\prime}\right)\right. \\
& \left.+\frac{\mathrm{i}}{2} \int d^{5} x\left(\bar{J}(x) \Psi_{J}(x)+\bar{\Psi}_{J}(x) J(x)\right)\right\} .
\end{aligned}
$$

Here we have changed the integration variables from $\Psi$ to $\Psi^{\prime}$, for which the Jacobian is unity. Denoting the integration over $\Psi^{\prime}$ and $\bar{\Psi}^{\prime}$ by $Z_{0}[0]$, we observe that the generating functional becomes

$$
Z_{0}[J, \bar{J}]=Z_{0}[0] \exp \left\{\frac{\mathrm{i}}{2} \int d^{5} x\left(\bar{J}(x) \Psi_{J}(x)+\bar{\Psi}_{J}(x) J(x)\right)\right\} .
$$

The field $\Psi_{J}(x)$ can be written as

$$
\Psi_{J}(x)=-\int d^{5} y S_{1}(x-y) J(y),
$$

where $S_{1}(x-y)$ is the free-field Green's function, which satisfies

$$
\left(\mathrm{i} \gamma^{\mu} \partial_{\mu}-k\right) S_{1}(x-y)=\tilde{\delta}^{5}(x-y)
$$

where we adopt a non-standard definition of the delta function:

$$
\tilde{\delta}^{5}(x-y) \equiv \tilde{\delta}^{5}(x-y ; m)+\tilde{\delta}^{5}(x-y ;-m),
$$

with $\tilde{\delta}^{5}(x-y ; \pm m)$ being Dirac delta functions [5] in $\mathcal{G}_{(4+1)}$ defined as

$$
\tilde{\delta}^{5}(x-y ; \pm m)=e^{\mp \mathrm{i} m \bar{c}\left(x^{5}-y^{5}\right)} \delta^{3}(\mathbf{x}-\mathbf{y}) \delta\left(x^{4}-y^{4}\right) .
$$

Taking $S_{1}(x-y)$ as

$$
S_{1}(x-y)=-\frac{\mathrm{i} \gamma^{\mu} \partial_{\mu}+k}{2 k} \Delta(x-y),
$$

and introducing the i $\varepsilon$ prescription by replacing $k^{2}$ with $k^{2}-\mathrm{i} \varepsilon$, we bring Eq. (19) into the form

$$
\frac{1}{2 k}\left(\partial_{\mu} \partial^{\mu}+k^{2}-\mathrm{i} \varepsilon\right) \Delta(x-y)=\tilde{\delta}^{5}(x-y),
$$

which is the equation for the Feynman propagator for a free Galilean scalar field. In the next section, we will show that $\Delta(x-y)$ coincides with the Galilean Feynman propagator up to a constant factor.

The equation for the adjoint field $\bar{\Psi}_{J}(x)$,

$$
\bar{\Psi}_{J}(x)\left(\mathrm{i} \gamma^{\mu} \overleftarrow{\partial}_{\mu}+k\right)=\bar{J}(x)
$$


is solved by

$$
\bar{\Psi}_{J}(x)=-\int d^{5} y \bar{J}(y) S_{2}(x-y),
$$

where $S_{2}(x-y)$ is a solution of

$$
S_{2}(x-y)\left(\mathrm{i} \gamma^{\mu} \overleftarrow{\partial}_{\mu}+k\right)=-\tilde{\delta}^{5}(x-y)
$$

The function $S_{2}(x-y)$ is related to $\Delta(x-y)$ by

$$
S_{2}(x-y)=\frac{1}{2 k} \Delta(x-y)\left(\mathrm{i} \gamma^{\mu} \overleftarrow{\partial}_{\mu}-k\right)
$$

so that

$$
S_{2}(y-x)=S_{1}(x-y) .
$$

For the external source $J$, we factor out the coordinate $x^{5}$ as follows :

$$
J(x)=e^{-\mathrm{i} m \bar{c} x^{5}} j_{+}\left(\mathbf{x}, x^{4}\right)+e^{\mathrm{i} m \bar{c} x^{5}} j_{-}\left(\mathbf{x}, x^{4}\right),
$$

and similarly for $\bar{J}(x)$. This factorization is motivated by the definition of the fields $\Psi_{J}(x)$ and $\bar{\Psi}_{J}(x)$ in Eqs. (18) and (23), respectively, so that we have, for instance :

$$
\begin{aligned}
\left(\mathrm{i} \gamma^{\mu} \partial_{\mu}-k\right) \Psi_{J}(x)= & -\int d^{5} y \tilde{\delta}^{5}(x-y) J(y), \\
= & \lim _{l \rightarrow \infty} \frac{1}{l} \int_{-l / 2}^{l / 2} d y^{5}\left[e^{-\mathrm{i} m \bar{c}\left(x^{5}-y^{5}\right)}+e^{\mathrm{i} m \bar{c}\left(x^{5}-y^{5}\right)}\right] \times \\
& \quad \times\left[e^{-\mathrm{i} m \bar{c} y^{5}} j_{+}\left(\mathbf{x}, x^{4}\right)+e^{\mathrm{i} m \bar{c} y^{5}} j_{-}\left(\mathbf{x}, x^{4}\right)\right] \\
= & -J(x),
\end{aligned}
$$

in agreement with Eq. (17). Note that in the second line, we have eliminated $y$ by integration and by using the definition of the delta function given in Eq. (20).

With the fields $\Psi_{J}(x)$ and $\bar{\Psi}_{J}(x)$ defined by Eqs. (18) and (23) respectively, the generating functional takes the form

$$
Z_{0}[J, \bar{J}]=Z_{0}[0] \exp \left\{-\mathrm{i} \int d^{5} x d^{5} y \bar{J}(x) S_{1}(x-y) J(y)\right\} .
$$

This is the generating functional of the Green's function that characterizes the Dirac field. Note that $Z_{0}[J, \bar{J}]$ is written in terms of the propagator $S_{1}(x-y)$, which includes both particle and antiparticle contributions.

\section{Green's functions for particles and antiparticles}

Let us now turn to some properties of the Galilean propagators $\Delta(x-y)$ and $S_{1}(x-y)$. The Fourier transforms of these propagators are defined by expressions similar to the mass-shell condition:

$$
\begin{aligned}
\Delta(x-y) & =\frac{1}{(2 \pi)^{5}} \int d^{5} p \bar{\Delta}(p) e^{-\mathrm{i} p(x-y)} 2 \pi\left[\delta\left(p^{4}-m \bar{c}\right)+\delta\left(p^{4}+m \bar{c}\right)\right] \\
S_{1}(x-y) & =\frac{1}{(2 \pi)^{5}} \int d^{5} p \bar{S}_{1}(p) e^{-\mathrm{i} p(x-y)} 2 \pi\left[\delta\left(p^{4}-m \bar{c}\right)+\delta\left(p^{4}+m \bar{c}\right)\right] .
\end{aligned}
$$


By substituting Eq. (25) into Eq. (22) and using

$$
\tilde{\delta}^{5}(x-y)=\frac{1}{(2 \pi)^{5}} \int d^{5} p e^{-\mathrm{i} p(x-y)} 2 \pi\left[\delta\left(p^{4}-m \bar{c}\right)+\delta\left(p^{4}+m \bar{c}\right)\right],
$$

where the term between brackets is reminiscent of our non-standard definition of delta function in $(4+1)$ dimensions, we find

$$
\bar{\Delta}(p)=\frac{-2 k}{p_{\mu} p^{\mu}-k^{2}+\mathrm{i} \varepsilon},
$$

so that we rewrite $\Delta(x-y)$ as

$$
\Delta(x-y)=\frac{\sqrt{2}}{(2 \pi)^{4}} \int d^{3} \mathbf{p} \int d p^{5} e^{\mathrm{i} \mathbf{p}(\mathbf{x}-\mathbf{y})-\mathrm{i} p^{5}\left(x^{4}-y^{4}\right)}\left[\frac{e^{\mathrm{i} m \bar{c}\left(x^{5}-y^{5}\right)}}{p^{5}+\frac{E}{\bar{c}}-\mathrm{i} \frac{\varepsilon}{2 m \bar{c}}}-\frac{e^{-\mathrm{i} m \bar{c}\left(x^{5}-y^{5}\right)}}{p^{5}-\frac{E}{\bar{c}}+\mathrm{i} \frac{\varepsilon}{2 m \bar{c}}}\right] .
$$

Integrating over $p^{5}$ with the change of variable $p^{5} \rightarrow p^{5}+\frac{E}{\bar{c}}$ in the first integral, and $p^{5} \rightarrow p^{5}-\frac{E}{\bar{c}}$ in the second one, and by using the following representation of the step function :

$$
\theta(\tau)=\lim _{\varepsilon \rightarrow 0^{+}} \frac{-1}{2 \pi \mathrm{i}} \int_{-\infty}^{\infty} d \omega \frac{e^{-\mathrm{i} \omega \tau}}{\omega+\mathrm{i} \varepsilon}
$$

we obtain

$$
\Delta(x-y)=\sqrt{2} \Delta_{F}(x-y),
$$

where

$$
-i \Delta_{F}(x-y) \equiv \theta\left(x^{4}-y^{4}\right) \Delta(x-y ; m)+\theta\left(y^{4}-x^{4}\right) \Delta(x-y ;-m),
$$

$\Delta_{F}(x-y)$ being the Galilean Feynman propagator for a free scalar field [20], and

$$
\Delta(x-y ; \pm m)=\frac{1}{(2 \pi)^{3}} \int d^{3} \mathbf{p} e^{\mp \mathrm{i} p(x-y)} .
$$

The positive- and negative-energy/mass contributions to $\Delta(x-y)$ can be written explicitly as

$$
\begin{aligned}
& \Delta(x-y)=\sqrt{2}\left[e^{-\mathrm{i} m \bar{c}\left(x^{5}-y^{5}\right)} G_{+}^{0}\left(\mathbf{x}-\mathbf{y} ; x^{4}-y^{4} ; m\right)\right. \\
& \left.+e^{\mathrm{i} m \bar{c}\left(x^{5}-y^{5}\right)} G_{-}^{0}\left(\mathbf{x}-\mathbf{y} ; x^{4}-y^{4} ;-m\right)\right],
\end{aligned}
$$

where

$$
G_{+}^{0}\left(\mathbf{x}-\mathbf{y} ; x^{4}-y^{4} ; m\right) \equiv \frac{\mathrm{i}}{(2 \pi)^{3}} \theta\left(x^{4}-y^{4}\right) \int d^{3} \mathbf{p} e^{\mathrm{i} \mathbf{p}(\mathbf{x}-\mathbf{y})-\mathrm{i} \frac{E}{\bar{c}}\left(x^{4}-y^{4}\right)}
$$

is the Schrödinger Green's function of a scalar particle with mass $m$ [5], and

$$
G_{-}^{0}\left(\mathbf{x}-\mathbf{y} ; x^{4}-y^{4} ;-m\right)=G_{+}^{0}\left(\mathbf{y}-\mathbf{x} ; y^{4}-x^{4} ; m\right) .
$$


This ensures that Eq. (27) is compatible with our earlier statements about the splitting of positive versus negative energy and mass.

With Eq. (27), $S_{1}(x-y)$ becomes

$$
S_{1}(x-y)=\sqrt{2} S_{F}(x-y),
$$

where

$$
S_{F}(x-y) \equiv-\frac{1}{2 k}\left(i \gamma^{\mu} \partial_{\mu}+k\right) \Delta_{F}(x-y)
$$

is the Galilean Feynman propagator for a free Dirac field. If we substitute Eqs. (25) and (26) into Eq. (21), we find

$$
\bar{S}_{1}(p)=\frac{\gamma^{\mu} p_{\mu}+k}{p_{\mu} p^{\mu}-k^{2}+\mathrm{i} \varepsilon} .
$$

Using Eq. (27), we write $S_{1}(x-y)$ in the form :

$$
S_{1}(x-y)=e^{-\mathrm{i} m \bar{c}\left(x^{5}-y^{5}\right)} S_{1}\left(\mathbf{x}-\mathbf{y}, x^{4}-y^{4} ; m\right)+e^{\mathrm{i} m \bar{c}\left(x^{5}-y^{5}\right)} S_{1}\left(\mathbf{x}-\mathbf{y}, x^{4}-y^{4} ;-m\right),
$$

where

$$
S_{1}\left(\mathbf{x}-\mathbf{y}, x^{4}-y^{4} ; \pm m\right)=\sqrt{2} S_{ \pm}^{0}\left(\mathbf{x}-\mathbf{y} ; x^{4}-y^{4} ; \pm m\right)
$$

and

$$
\begin{aligned}
S_{+}^{0}\left(\mathbf{x}-\mathbf{y}, x^{4}-y^{4} ; m\right) & =\frac{1}{2 k} \gamma^{4} \delta\left(x^{4}-y^{4}\right) \delta(\mathbf{x}-\mathbf{y}) \\
& -\theta\left(x^{4}-y^{4}\right) \frac{\mathrm{i}}{(2 \pi)^{3}} \int d^{3} \mathbf{p} S_{+}(\mathbf{p}, m) e^{\mathrm{i}\left[\mathbf{p}(\mathbf{x}-\mathbf{y})-\frac{E}{\bar{c}}\left(x^{4}-y^{4}\right)\right]}
\end{aligned}
$$

is the Schrödinger Green's function of a Dirac particle of mass $m$ with

$$
S_{+}(\mathbf{p}, m)=\frac{1}{4 E_{k}}\left(\begin{array}{cc}
E+3 E_{k} & E-E_{k}-\mathrm{i} \bar{c} \sqrt{2} \sigma \cdot \mathbf{p} \\
-E+E_{k}-\mathrm{i} \bar{c} \sqrt{2} \sigma \cdot \mathbf{p} & -E+E_{k}
\end{array}\right) .
$$

For the negative-mass contribution, we have

$$
\begin{aligned}
S_{-}^{0}\left(\mathbf{x}-\mathbf{y}, x^{4}-y^{4} ;-m\right) & =-\frac{1}{2 k} \gamma^{4} \delta\left(x^{4}-y^{4}\right) \delta(\mathbf{x}-\mathbf{y}) \\
& -\theta\left(y^{4}-x^{4}\right) \frac{\mathrm{i}}{(2 \pi)^{3}} \int d^{3} \mathbf{p} S_{-}(\mathbf{p}, m) e^{-\mathrm{i}\left[\mathbf{p}(\mathbf{x}-\mathbf{y})-\frac{E}{\bar{c}}\left(x^{4}-y^{4}\right)\right]}
\end{aligned}
$$

where

$$
S_{-}(\mathbf{p}, m)=1-S_{+}(\mathbf{p}, m) .
$$

The propagator $S_{F}(x-y)$ can be defined in the canonical formalism as well. The expansions in Eqs. (15) and (16) yield an expression for the Feynman propagator as

$$
\langle 0|T[\Psi(x) \bar{\Psi}(y)]| 0\rangle=i S_{F}(x-y),
$$


where $T$ denotes the time ordering. This formula connects the path-integral and canonical formalism and proves their equivalence.

Now let us define the Galilean one-particle Green's function for free fields :

$$
G^{0}\left(x_{1}, x_{2}\right)=\left.\frac{(-\mathrm{i})^{2}}{Z_{0}[0]} \frac{\delta^{2} Z_{0}[J, \bar{J}]}{\delta \bar{J}\left(x_{1}\right) \delta J\left(x_{2}\right)}\right|_{J=0=\bar{J}}
$$

where $Z_{0}[J, \bar{J}]$ is given in Eq. (24), thus leading to

$$
G^{0}\left(x_{1}, x_{2}\right)=-\mathrm{i} \sqrt{2} S_{F}\left(x_{1}-x_{2}\right) .
$$

It is possible to calculate the average values of the translation generators in the Hilbert space given in Eq. (3), i.e. the observables corresponding to momentum, energy and mass in quantum mechanics :

$$
\langle\widehat{\mathcal{O}}\rangle=\mathrm{i} \int d^{3} x d x^{5} \lim _{y \rightarrow x}\left[\mathcal{O} G^{0}(x, y)\right]
$$

where $\mathcal{O}$ denotes $P_{i}, H=P_{4}$ or $M=P_{5}$.

We may generalize the one-particle Green's function in Eq. (29) to the $n$-particle Green's functions :

$$
\begin{aligned}
G^{0}\left(x_{1}, \ldots, x_{n} ; y_{1}, \ldots, y_{n}\right) & =\langle 0| T\left(\Psi\left(x_{1}\right) \cdots \Psi\left(x_{n}\right) \bar{\Psi}\left(y_{1}\right) \cdots \bar{\Psi}\left(y_{n}\right)|0\rangle\right. \\
& =\left.\frac{(-\mathrm{i})^{2 n}}{Z_{0}[0]} \frac{\delta^{2 n} Z_{0}[J, \bar{J}]}{\delta \bar{J}\left(x_{1}\right) \ldots \delta \bar{J}\left(x_{n}\right) \delta J\left(y_{1}\right) \ldots \delta J\left(y_{n}\right)}\right|_{J=0=\bar{J}}
\end{aligned}
$$

For instance, the 1-particle Green's function is given in Eq. (29) and the 2-particle Green's function is given as

$$
G^{0}\left(x_{1}, x_{2} ; y_{1}, y_{2}\right)=\left.\frac{1}{Z_{0}[0]} \frac{\delta^{4} Z_{0}[J, \bar{J}]}{\delta \bar{J}\left(x_{1}\right) \delta \bar{J}\left(x_{2}\right) \delta J\left(y_{1}\right) \delta J\left(y_{2}\right)}\right|_{J=0=\bar{J}} .
$$

Explicit forms and perturbative expansion will be given later on in an interacting system with quartic interactions. For one-particle Green's function, an equation similar to the Schwinger-Dyson equation is obtained with the self-energy defined explicitly.

\section{Self-interacting quartic potential}

Now consider a Lagrangian which contains a non-trivial interacting potential :

$$
\mathcal{L}=\mathcal{L}_{0}+\mathcal{L}_{\text {int }}
$$


where $\mathcal{L}_{0}$ is given by Eq. (6) and $\mathcal{L}_{\text {int }}$ is the interaction term that depends on $\Psi$ and $\bar{\Psi}$. With an arbitrary interaction, the generating functional is

$$
Z[J, \bar{J}]=\frac{\int \mathcal{D} \Psi \int \mathcal{D} \bar{\Psi} \exp \left\{\mathrm{i} I-\mathrm{i} \int d^{5} x[J(x) \bar{\Psi}(x)+\bar{J}(x) \Psi(x)]\right\}}{\int \mathcal{D} \Psi \int \mathcal{D} \bar{\Psi} \exp (\mathrm{i} I)}
$$

with $I$ given in Eq. $(7)$, and where $\mathcal{L}_{0}$ is replaced by $\mathcal{L}$. Following standard methods [24], we write the generating functional as

$$
Z[J, \bar{J}]=N \exp \left\{-\mathrm{i} \int d^{5} x \mathcal{L}_{\text {int }}\left[\frac{1}{\mathrm{i}} \frac{\delta}{\delta \bar{J}}, \frac{1}{\mathrm{i}} \frac{\delta}{\delta J}\right]\right\} Z_{0}[J, \bar{J}]
$$

where $N$ is a normalization factor, and $Z_{0}$ is given by Eq. (24). We derive the Green's functions from Eq. (31) by replacing $Z_{0}[J, \bar{J}]$ with $Z[J, \bar{J}]$.

Consider an interaction Lagrangian in the form

$$
\mathcal{L}_{\text {int }}=g(\bar{\Psi}(x) \Psi(x))^{2} .
$$

When we expand Eq. (33) in powers of $g$, then $Z[J, \bar{J}]$ becomes

$$
\begin{aligned}
Z[J, \bar{J}]= & N \exp \left\{-\mathrm{i} g \int d^{5} x\left[\frac{\delta}{\delta J(x)} \frac{\delta}{\delta \bar{J}(x)} \frac{\delta}{\delta J(x)} \frac{\delta}{\delta \bar{J}(x)}\right]\right\} Z_{0}[J, \bar{J}] \\
=N & \left\{1-\mathrm{i} g \int d^{5} z\left(\frac{\delta}{\delta J(z)} \frac{\delta}{\delta \bar{J}(z)} \frac{\delta}{\delta J(z)} \frac{\delta}{\delta \bar{J}(z)}\right)\right. \\
& -\frac{g^{2}}{2} \int d^{5} z d^{5} w\left(\frac{\delta}{\delta J(w)} \frac{\delta}{\delta \bar{J}(w)} \frac{\delta}{\delta J(w)} \frac{\delta}{\delta \bar{J}(w)}\right)\left(\frac{\delta}{\delta J(z)} \frac{\delta}{\delta \bar{J}(z)} \frac{\delta}{\delta J(z)} \frac{\delta}{\delta \bar{J}(z)}\right)+ \\
& \left.+O\left(g^{3}\right)\right\} Z_{0}[J, \bar{J}] .
\end{aligned}
$$

To zeroth order in $g$, we simply retrieve $Z_{0}[J, \bar{J}]$.

Henceforth, we shall utilize the short-hand notation

$$
\left\langle\bar{J}_{\alpha}(x) S_{\alpha \beta}(x-y) J_{\beta}(y)\right\rangle \equiv \int d^{5} x d^{5} y \bar{J}_{\alpha}(x) S_{\alpha \beta}(x-y) J_{\beta}(y)
$$

where $S_{\alpha \beta}(x-y) \equiv\left(S_{1}\right)_{\alpha \beta}(x-y)$, and $\alpha, \beta=1,2,3,4$ indicate the component structure of virtual sources. Thus we have

$$
Z_{0}=e^{-\mathrm{i}\left\langle\bar{J}_{\alpha}(x) S_{\alpha \beta}(x-y) J_{\beta}(y)\right\rangle} .
$$

To second order in $g$, we find

$$
Z[J, \bar{J}]=N\left\{1-\mathrm{i} g \int d^{5} z T(z)-\frac{g^{2}}{2} \int d^{5} z d^{5} w T(z, w)\right\} Z_{0}[J, \bar{J}],
$$

where

$$
T(z) \equiv \frac{1}{Z_{0}[J, \bar{J}]} \frac{\delta}{\delta J_{\alpha}(z)} \frac{\delta}{\delta \bar{J}_{\alpha}(z)} \frac{\delta}{\delta J_{\beta}(z)} \frac{\delta}{\delta \bar{J}_{\beta}(z)} Z_{0}[J, \bar{J}]=\operatorname{Tr}\left(S_{1}(0)\right)^{2}-\left(\operatorname{Tr} S_{1}(0)\right)^{2}
$$




$$
\begin{gathered}
-2 \mathrm{i}\left(S_{\alpha \beta}(0)-\delta_{\alpha \beta} \operatorname{Tr} S_{1}(0)\right)\left\langle S_{\beta \delta}(z-v) J_{\delta}(v)\right\rangle\left\langle\bar{J}_{\gamma}(u) S_{\gamma \alpha}(u-z)\right\rangle \\
+\left\langle S_{\beta \delta}(z-v) J_{\delta}(v)\right\rangle\left\langle\bar{J}_{\gamma}(u) S_{\gamma \beta}(u-z)\right\rangle\left\langle S_{\alpha \bar{\delta}}(z-w) J_{\bar{\delta}}(w)\right\rangle\left\langle\bar{J}_{\bar{\gamma}}(y) S_{\bar{\gamma} \alpha}(y-z)\right\rangle,
\end{gathered}
$$

and

$$
T(z, w) \equiv \frac{1}{Z_{0}[J, \bar{J}]} \frac{\delta}{\delta J_{\alpha}(w)} \frac{\delta}{\delta \bar{J}_{\alpha}(w)} \frac{\delta}{\delta J_{\beta}(w)} \frac{\delta}{\delta \bar{J}_{\beta}(w)}\left(T(z) Z_{0}[J, \bar{J}]\right)
$$

The normalization factor in Eq. (34) is chosen in such way that $Z[0]=1$,

$$
N=Z_{0}^{-1}[0]\left(1-\mathrm{i} g \int d^{5} z T_{0}(z)-\frac{g^{2}}{2} \int d^{5} z d^{5} w T_{0}(z, w)\right)^{-1},
$$

where

$$
\left.T_{0}(z) \equiv T(z)\right|_{J=0=\bar{J}},\left.\quad T_{0}(z, w) \equiv T(z, w)\right|_{J=0=\bar{J}}
$$

excluding vacuum graphs from consideration.

\subsection{2-point function}

The 2-point function $G\left(x_{1}, x_{2}\right)$ is given by Eq. (29) with $Z_{0}[J, \bar{J}]$ replaced by $Z[J, \bar{J}]$. To zero-th order in $g, G\left(x_{1}, x_{2}\right)$ is clearly the same as for the free field. To first order in $g$, we obtain

$$
G_{\alpha \beta}\left(x_{1}, x_{2}\right)=G_{\alpha \beta}^{0}\left(x_{1}, x_{2}\right)-2 g \int d^{5} z G_{\alpha \bar{\alpha}}^{0}\left(x_{1}, z\right)\left(S_{\bar{\alpha} \bar{\beta}}(0)-\delta_{\bar{\alpha} \bar{\beta}} \operatorname{Tr} S_{1}(0)\right) G_{\bar{\beta} \beta}^{0}\left(z, x_{2}\right) .
$$

Using the definition of $S_{1}(x-y)$ given in Eq. (26), yields the positive and negativemass contributions to $S_{\bar{\alpha} \bar{\beta}}(0)$,

$$
S_{\bar{\alpha} \bar{\beta}}(0 ; \pm m)=\frac{1}{(2 \pi)^{3}} \int d^{3} \mathbf{p} \int d p^{5} \frac{\left[\frac{1}{2 m c}\left(\gamma^{4} p^{5}+k\right) \pm \frac{1}{2} \gamma^{5}\right]_{\bar{\alpha} \bar{\beta}}}{ \pm p^{5}-\frac{E}{c}+\mathrm{i} \frac{\varepsilon}{2 m \bar{c}}}
$$

Taking into account the identity,

$$
\frac{1}{\omega \pm i \varepsilon}=\mathcal{P} \frac{1}{\omega} \mp \mathrm{i} \pi \delta(\omega)
$$

where $\varepsilon>0$ and $\mathcal{P}$ denotes the principal value, non-diagonal elements of $S_{\bar{\alpha} \bar{\beta}}(0 ; m)$ and $S_{\bar{\alpha} \bar{\beta}}(0 ;-m)$ can be shown to be equal in magnitude and opposite in sign, so that they cancel each other, and $S_{\bar{\alpha} \bar{\beta}}(0)=S_{\bar{\alpha} \bar{\beta}}(0 ; m)+S_{\bar{\alpha} \bar{\beta}}(0 ;-m)$ is diagonal,

$$
S_{\bar{\alpha} \bar{\beta}}(0)=\frac{1}{4} \delta_{\bar{\alpha} \bar{\beta}} \operatorname{Tr} S_{1}(0)
$$



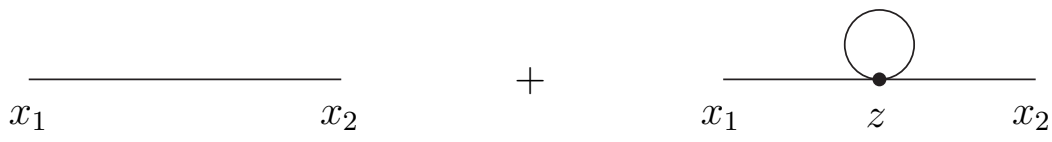

Figure 1: Diagrams for the first order 2-point function for the quartic potential

where

$$
\operatorname{Tr} S_{1}(0)=2 \operatorname{Tr} S_{1}(0 ; m)=-\frac{\mathrm{i}}{2 \sqrt{2} \pi^{3}} \int d^{3} \mathbf{p}
$$

is a divergent quantity, which can be made finite with a cutoff. This serious divergence problem may be resolved by including a momentum-dependant vertex function. For example, if the vertex function decreases rapidly with momentum, then this integral can be convergent and finite. Such a form may be anticipated for any realistic formulation of the problem.

With Eq. (36), the first order 2-point function can be rewritten as

$$
G_{\alpha \beta}\left(x_{1}, x_{2}\right)=G_{\alpha \beta}^{0}\left(x_{1}, x_{2}\right)+G_{\alpha \beta}^{(1)}\left(x_{1}, x_{2}\right),
$$

where

$$
G_{\alpha \beta}^{(1)}\left(x_{1}, x_{2}\right) \equiv \Sigma_{1}(0) \int d^{5} z G_{\alpha \bar{\alpha}}^{0}\left(x_{1}, z\right) G_{\bar{\alpha} \beta}^{0}\left(z, x_{2}\right)
$$

and

$$
\Sigma_{1}(0) \equiv \frac{3 g}{2} \operatorname{Tr} S_{1}(0),
$$

and represented diagrammatically in Fig. 1. The first diagram shows the 2-point function for a free Galilean Dirac field. In the second diagram, $\Sigma_{1}(0)$ is represented by a closed loop with one vertex on it.

The second order in g contribution to the 2-point function is

$$
G_{\alpha \beta}^{(2)}\left(x_{1}, x_{2}\right)=\left.\frac{g^{2}}{2} \int d^{5} z d^{5} w \frac{\delta^{2}}{\delta \bar{J}_{\alpha}\left(x_{1}\right) \delta J_{\beta}\left(x_{2}\right)}\left(T(z, w)-2 T_{0}(z) T(w)\right)\right|_{J=0=\bar{J}},
$$

that is the sum of three terms

$$
\begin{aligned}
G_{\alpha \beta}^{(2)}\left(x_{1}, x_{2}\right) & =g^{2} \int d^{5} z d^{5} w\left[4 G_{\alpha \delta}^{0}\left(x_{1}, w\right) G_{\delta \bar{\alpha}}^{0}(w, z) \Gamma_{\bar{\alpha} \bar{\beta}}(z, w) G_{\bar{\beta} \beta}^{0}\left(z, x_{2}\right)\right. \\
& +3 \mathrm{i} \operatorname{Tr} S_{1}(0) \cdot G_{\alpha \bar{\alpha}}^{0}\left(x_{1}, z\right) \Gamma_{\bar{\alpha} \bar{\beta}}(z, w) G_{\bar{\beta} \beta}^{0}\left(z, x_{2}\right) \\
& \left.+\frac{9}{4}\left(\operatorname{Tr} S_{1}(0)\right)^{2} \cdot G_{\alpha \bar{\alpha}}^{0}\left(x_{1}, z\right) G_{\bar{\alpha} \bar{\beta}}^{0}(z, w) G_{\bar{\beta} \beta}^{0}\left(w, x_{2}\right)\right] .
\end{aligned}
$$

The corresponding diagrams are shown in Fig. 2. The function $\Gamma_{\bar{\alpha} \bar{\beta}}(z, w)$ in Eq. (37) is defined as

$$
\Gamma_{\bar{\alpha} \bar{\beta}}(z, w) \equiv-G_{\bar{\alpha} \delta}^{0}(z, w) G_{\delta \bar{\beta}}^{0}(w, z)+\delta_{\bar{\alpha} \bar{\beta}} G_{\gamma \delta}^{0}(z, w) G_{\delta \gamma}^{0}(w, z),
$$




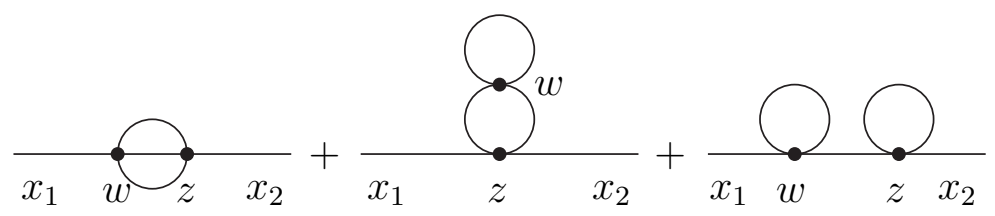

Figure 2: Diagrams for the $g^{2}$-order of the 2-point function for the quartic potential. The third diagram is not one-particle irreducible. The contribution of second diagram vanishes.

being represented by a closed loop with two vertices. Each of the vertices can have up to two external lines. However, for the first diagram in Fig. 2, one leg of the vertex at $w$ is joined to a leg of the vertex at $z$ creating an internal line, so that we have one external line at each vertex. At the second diagram, two legs of the vertex at $w$ are joined together, producing $\Sigma_{1}(0)$. From Eq. (38), we deduce the following relations for $\Gamma_{\bar{\alpha} \bar{\beta}}(z, w)$ :

$$
\Gamma_{\bar{\alpha} \bar{\alpha}}(z, w)=3 G_{\gamma \delta}^{0}(z, w) G_{\delta \gamma}^{0}(w, z),
$$

and

$$
\Gamma_{\bar{\alpha} \bar{\beta}}(z, z)=-\frac{3}{16} \delta_{\bar{\alpha} \bar{\beta}}\left(\operatorname{Tr} S_{1}(0)\right)^{2} .
$$

The second diagram in Fig. 1 and the first two in Fig. 2 are one-particle irreducible; they cannot be disconnected by cutting through any one internal line. The third diagram in Fig. 2 is a chain of two first-order one-particle irreducible graphs.

To simplify the expression for $G_{\alpha \beta}^{(2)}\left(x_{1}, x_{2}\right)$, Eq. (37), we can factor the fifth coordinate out of the functions $G^{0}$ and $\Gamma$ and then perform integrations over $z^{5}$ and $w^{5}$. The function $\Gamma_{\bar{\alpha} \bar{\beta}}(z, w)$ is factorized as follows

$$
\begin{gathered}
\Gamma_{\bar{\alpha} \bar{\beta}}(z, w)=\Gamma_{\bar{\alpha} \bar{\beta}}^{0}\left(\mathbf{z}, \mathbf{w}, z^{4}, w^{4} ; m\right)+e^{-2 \mathrm{i} m \bar{c}\left(z^{5}-w^{5}\right)} \Gamma_{\bar{\alpha} \bar{\beta}}^{(+)}\left(\mathbf{z}, \mathbf{w}, z^{4}, w^{4} ; m\right)+ \\
+e^{2 \mathrm{i} m \bar{c}\left(z^{5}-w^{5}\right)} \Gamma_{\bar{\alpha} \bar{\beta}}^{(-)}\left(\mathbf{z}, \mathbf{w}, z^{4}, w^{4} ; m\right),
\end{gathered}
$$

where

$$
\begin{aligned}
\Gamma_{\bar{\alpha} \bar{\beta}}^{0}\left(\mathbf{z}, \mathbf{w}, z^{4}, w^{4} ; m\right) \equiv-[ & G_{\bar{\alpha} \delta}^{0}\left(\mathbf{z}, \mathbf{w}, z^{4}, w^{4} ; m\right) G_{\delta \bar{\beta}}^{0}\left(\mathbf{w}, \mathbf{z}, w^{4}, z^{4} ; m\right)+ \\
& \left.+G_{\bar{\alpha} \delta}^{0}\left(\mathbf{z}, \mathbf{w}, z^{4}, w^{4} ;-m\right) G_{\delta \bar{\beta}}^{0}\left(\mathbf{w}, \mathbf{z}, w^{4}, z^{4} ;-m\right)\right]+ \\
+ & \delta_{\bar{\alpha} \bar{\beta}}\left[G_{\gamma \delta}^{0}\left(\mathbf{z}, \mathbf{w}, z^{4}, w^{4} ; m\right) G_{\delta \gamma}^{0}\left(\mathbf{w}, \mathbf{z}, w^{4}, z^{4} ; m\right)+\right. \\
+ & \left.G_{\gamma \delta}^{0}\left(\mathbf{z}, \mathbf{w}, z^{4}, w^{4} ;-m\right) G_{\delta \gamma}^{0}\left(\mathbf{w}, \mathbf{z}, w^{4}, z^{4} ;-m\right)\right]
\end{aligned}
$$


is that part of $\Gamma_{\bar{\alpha} \bar{\beta}}(z, w)$, which does not oscillate in the fifth coordinates, while

$$
\begin{aligned}
\Gamma_{\bar{\alpha} \bar{\beta}}^{(+)}\left(\mathbf{z}, \mathbf{w}, z^{4}, w^{4} ; m\right) \equiv- & -G_{\bar{\alpha} \delta}^{0}\left(\mathbf{z}, \mathbf{w}, z^{4}, w^{4} ; m\right) G_{\delta \bar{\beta}}^{0}\left(\mathbf{w}, \mathbf{z}, w^{4}, z^{4} ;-m\right)+ \\
& +\delta_{\bar{\alpha} \bar{\beta}} G_{\gamma \delta}^{0}\left(\mathbf{z}, \mathbf{w}, z^{4}, w^{4} ; m\right) G_{\delta \gamma}^{0}\left(\mathbf{w}, \mathbf{z}, w^{4}, z^{4} ;-m\right)
\end{aligned}
$$

and

$$
\Gamma_{\bar{\alpha} \bar{\beta}}^{(-)}\left(\mathbf{z}, \mathbf{w}, z^{4}, w^{4} ; m\right) \equiv \Gamma_{\bar{\alpha} \bar{\beta}}^{(+)}\left(\mathbf{z}, \mathbf{w}, z^{4}, w^{4} ;-m\right) .
$$

Integrating both parts of Eq. (39) over $w^{5}$ (or $z^{5}$ ) and using the limit

$$
\lim _{l \rightarrow \infty} \frac{1}{l} \int_{-l / 2}^{l / 2} d w^{5} e^{ \pm 2 i m \bar{c} w^{5}}=\lim _{l \rightarrow \infty} \frac{\sin (2 m \bar{c} l)}{m \bar{c} l}=0
$$

valid for nonzero values of $m$, we obtain

$$
\int d w^{5} \Gamma_{\bar{\alpha} \bar{\beta}}(z, w)=\Gamma_{\bar{\alpha} \bar{\beta}}^{0}\left(\mathbf{z}, \mathbf{w}, z^{4}, w^{4} ; m\right),
$$

i.e. the oscillating parts of $\Gamma_{\bar{\alpha} \bar{\beta}}(z, w)$ do not contribute to the integral.

Using the expressions given by Eqs. (28) and (39), we find that the product

$$
G_{\alpha \delta}^{0}\left(x_{1}, w\right) G_{\delta \bar{\alpha}}^{0}(w, z) \Gamma_{\bar{\alpha} \bar{\beta}}(z, w) G_{\bar{\beta} \beta}^{0}\left(z, x_{2}\right)
$$

in the first term of the right-hand side of Eq. (37) has the following non-oscillating parts in $z^{5}$ and $w^{5}$ :

$$
\begin{aligned}
& e^{-\mathrm{i} m \bar{c}\left(x_{1}^{5}-x_{2}^{5}\right)} G_{\alpha \delta}^{0}\left(\mathbf{x}_{1}, \mathbf{w}, x_{1}^{4}, w^{4} ; m\right) G_{\delta \bar{\alpha}}^{0}\left(\mathbf{w}, \mathbf{z}, w^{4}, z^{4} ; m\right) \times \\
& \quad \times \Gamma_{\bar{\alpha} \bar{\beta}}^{0}\left(\mathbf{z}, \mathbf{w}, z^{4}, w^{4} ; m\right) G_{\bar{\beta} \beta}^{0}\left(\mathbf{z}, \mathbf{x}_{2}, z^{4}, x_{2}^{4} ; m\right)+ \\
& +e^{i m \bar{c}\left(x_{1}^{5}-x_{2}^{5}\right)} G_{\alpha \delta}^{0}\left(\mathbf{x}_{1}, \mathbf{w}, x_{1}^{4}, w^{4} ;-m\right) G_{\delta \bar{\alpha}}^{0}\left(\mathbf{w}, \mathbf{z}, w^{4}, z^{4} ;-m\right) \times \\
& \quad \times \Gamma_{\bar{\alpha} \bar{\beta}}^{0}\left(\mathbf{z}, \mathbf{w}, z^{4}, w^{4} ;-m\right) G_{\bar{\beta} \beta}^{0}\left(\mathbf{z}, \mathbf{x}_{2}, z^{4}, x_{2}^{4} ;-m\right),
\end{aligned}
$$

so that the corresponding integral can be written as sum of positive- and negativemass contributions. Let us represent $G_{\alpha \delta}^{0}\left(\mathbf{x}_{1}, \mathbf{w}, x_{1}^{4}, w^{4} ; m\right)$ by a line in the $(3+1)$ space-time, with an arrow pointed in the direction in which the particle is moving, i.e. from $x_{1}$ to $w$, and $G_{\alpha \delta}^{0}\left(\mathbf{x}_{1}, \mathbf{w}, x_{1}^{4}, w^{4} ;-m\right)$ by a line again running from $x_{1}$ to $w$ and carrying an arrow in the opposite direction, as in Fig. 3.

(a) $\quad G_{\alpha \delta}^{0}\left(\mathbf{x}_{1}, \mathbf{w}, x_{1}^{4}, w^{4} ; m\right):$

$$
\left(\mathbf{x}_{1}, \overrightarrow{\left.x_{1}^{4}\right) \longrightarrow(\mathbf{w},} w^{4}\right)
$$

$$
G_{\alpha \delta}^{0}\left(\mathbf{x}_{1}, \mathbf{w}, x_{1}^{4}, w^{4} ;-m\right): \quad\left(\mathbf{x}_{1}, \overline{\left.x_{1}^{4}\right) \longleftarrow\left(\mathbf{w}, w^{4}\right)}\right.
$$

Figure 3: Green's functions in $(3+1)$ dimensions for (a) positive and (b) negative energy/mass 
When the Green's function $G_{\alpha \beta}^{0}$, defined in $(4+1)$-dimensions, is reduced to $(3+1)$ Galilean space-time, it contains two parts, one for positive energy and the other for negative energy. If we represent the $(4+1)$-dimensional Green's function by a simple line, and the $(3+1)$-dimensional Green's functions by a line containing an arrow, then the relation between the $(4+1)$-dimensional Green's function and the positiveand negative-energy/mass contributions in $(3+1)$ space-time diagrams is represented as in Fig. 4. The symmetry of the two-point Green's function under interchange of $x_{1}$ and $w$ is obvious.

$$
\begin{aligned}
G_{\alpha \beta}^{0}\left(x_{1}, w\right) & =x_{1} \\
& =e^{-\mathrm{i} m\left(x_{1}^{5}-w^{5}\right)} \underset{\left(\mathbf{x}_{1}, x_{1}^{4}\right) \quad\left(\mathbf{w}, w^{4}\right)}{\longrightarrow}+e^{+\mathrm{i} m\left(x_{1}^{5}-w^{5}\right)} \overline{\left(\mathbf{x}_{1}, x_{1}^{4}\right) \quad\left(\mathbf{w}, w^{4}\right)}
\end{aligned}
$$

Figure 4: Positive- and negative-energy/mass contributions to the Green's function through dimensional reduction

For instance, the total contribution of the first one-particle-irreducible secondorder diagram (in $(4+1)$ space-time) in Fig. 2 contains four diagrams after reduction to $(3+1)$ space-time. These diagrams are shown in Fig. 5. Although the number of diagrams increases after the reduction to $(3+1)$-dimensions, a clear interpretation in terms of particles and antiparticles becomes possible. The first two diagrams with external lines being particles represent the second-order positive-mass contribution to the 2-point function, while two others with external lines being antiparticles represent the second-order negative-mass contribution.
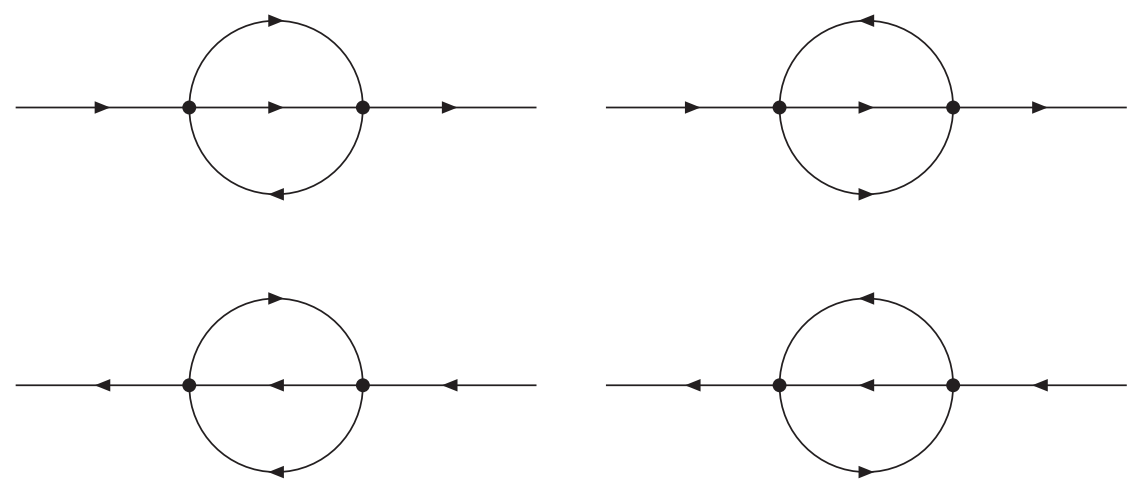
Figure 5: Contributions in $(3+1)$ space-time of the leftmost diagram in Fig. 2

Performing the integration over $w^{5}$ in the second term of the right-hand side of Eq. (37) as well and using Eq. (41), this gives

$$
\int d^{5} w \Gamma_{\bar{\alpha} \bar{\beta}}(z, w)=\int d^{3} \mathbf{w} \int d w^{4} \Gamma_{\bar{\alpha} \bar{\beta}}^{0}\left(\mathbf{z}, \mathbf{w}, z^{4}, w^{4} ; m\right) .
$$

It is clear from the expression for $\Gamma_{\bar{\alpha} \bar{\beta}}^{0}\left(\mathbf{z}, \mathbf{w}, z^{4}, w^{4} ; m\right)$, Eq. (40), that the positiveand negative- mass Green's functions contribute separately. Calculating these contributions, we have

$$
\int d^{3} \mathbf{w} \int d w^{4} G_{\bar{\alpha} \delta}^{0}\left(\mathbf{z}, \mathbf{w}, z^{4}, w^{4} ; \pm m\right) G_{\delta \bar{\beta}}^{0}\left(\mathbf{w}, \mathbf{z}, w^{4}, z^{4} ; \pm m\right)=\mp \frac{1}{8 m \bar{c}}\left(\gamma^{4}\right)_{\bar{\alpha} \bar{\beta}} \operatorname{Tr} S_{1}(0),
$$

that results in

$$
\int d^{3} \mathbf{w} \int d w^{4} \Gamma_{\bar{\alpha} \bar{\beta}}^{0}\left(\mathbf{z}, \mathbf{w}, z^{4}, w^{4} ; m\right)=0 .
$$

Therefore, the second diagram in Fig. 2 does not contribute.

Let us write the sum of one-particle-irreducible graphs for 2-point function in all orders in $g$ as

$$
\int d^{5} z d^{5} w G_{\alpha \bar{\alpha}}^{0}\left(x_{1}, z\right) \Sigma_{\bar{\alpha} \bar{\beta}}(z, w) G_{\bar{\beta} \beta}^{0}\left(w, x_{2}\right),
$$

with two external lines and one self-energy subgraph $\Sigma_{\bar{\alpha} \bar{\beta}}(z, w)$. To order $g^{2}$, we have

$$
\Sigma_{\bar{\alpha} \bar{\beta}}(z, w)=\delta_{\bar{\alpha} \bar{\beta}} \delta^{(5)}(z-w) \Sigma_{1}(0)+4 g^{2} G_{\bar{\alpha} \delta}^{0}(w, z) \Gamma_{\delta \bar{\beta}}(z, w)+O\left(g^{3}\right) .
$$

Then the complete 2-point function is given by a sum of chains of one, two, and more of these subgraphs connected with the free field propagators

$$
\begin{aligned}
& G_{\alpha \beta}\left(x_{1}, x_{2}\right)=G_{\alpha \beta}^{0}\left(x_{1}, x_{2}\right)+\int d^{5} z d^{5} w G_{\alpha \bar{\alpha}}^{0}\left(x_{1}, z\right) \Sigma_{\bar{\alpha} \bar{\beta}}(z, w) G_{\bar{\beta} \beta}^{0}\left(w, x_{2}\right)+ \\
& \quad+\int d^{5} z d^{5} w \int d^{5} \bar{z} d^{5} \bar{w} G_{\alpha \bar{\alpha}}^{0}\left(x_{1}, z\right) \Sigma_{\bar{\alpha} \bar{\beta}}(z, w) G_{\bar{\beta} \delta}^{0}(w, \bar{z}) \Sigma_{\delta \bar{\delta}}(\bar{z}, \bar{w}) G_{\bar{\delta} \beta}^{0}\left(\bar{w}, x_{2}\right)+\cdots \\
& \quad=G_{\alpha \beta}^{0}\left(x_{1}, x_{2}\right)+\int d^{5} z d^{5} w G_{\alpha \bar{\alpha}}^{0}\left(x_{1}, z\right) \Sigma_{\bar{\alpha} \bar{\beta}}(z, w) G_{\bar{\beta} \beta}\left(w, x_{2}\right) .
\end{aligned}
$$

Introducing the Fourier transform $\Sigma_{\alpha \beta}(p)$ and $G_{\alpha \beta}(p)$ of the functions $\Sigma_{\alpha \beta}\left(x_{1}, x_{2}\right)$ and $G_{\alpha \beta}\left(x_{1}, x_{2}\right)$ in the same way as the Fourier transforms of the Galilean propagators in equations (25) and (26), we rewrite Eq. $(42)$ in $(4+1)$-dimensional momentum space as

$$
\mathrm{i} G_{\alpha \beta}(p)=S_{\alpha \beta}(p)+S_{\alpha \bar{\alpha}}(p) \Sigma_{\bar{\alpha} \bar{\beta}}(p) G_{\bar{\beta} \beta}(p)
$$

that results in the exact expression for $G_{\alpha \beta}(p)$ :

$$
G_{\alpha \beta}(p)=\left[\mathrm{i} S_{1}^{-1}(p)-\Sigma(p)\right]_{\alpha \beta}^{-1} .
$$

This expression is similar to the case of many-body systems where the exact expression of the 2-point function depends on self-energy. 


\subsection{4-point function}

The 4-point function $G\left(x_{1}, x_{2} ; y_{1}, y_{2}\right)$ is given by Eq. (32) with $Z_{0}[J, \bar{J}]$ replaced by $Z[J, \bar{J}]$. To find its irreducible part, we can use a generating functional $W[J, \bar{J}]$, which generates only connected Feynman diagrams or connected Green's functions. It is related to $Z[J, \bar{J}]$ as

$$
W[J, \bar{J}]=-\mathrm{i} \ln Z[J, \bar{J}] .
$$

We define the irreducible or connected 4-point function as

$$
\bar{G}\left(x_{1}, x_{2} ; y_{1}, y_{2}\right)=\left.\frac{1}{W[0]} \frac{\delta^{4} W[J, \bar{J}]}{\delta \bar{J}\left(x_{1}\right) \delta \bar{J}\left(x_{2}\right) \delta J\left(y_{1}\right) \delta J\left(y_{2}\right)}\right|_{J=0=\bar{J}},
$$

that gives us the following relation between $\bar{G}\left(x_{1}, x_{2} ; y_{1}, y_{2}\right)$ and the complete 4-point function $G\left(x_{1}, x_{2} ; y_{1}, y_{2}\right)$ :

$$
\begin{aligned}
\bar{G}_{\alpha \beta \gamma \delta}\left(x_{1}, x_{2} ; y_{1}, y_{2}\right) & =-\mathrm{i} G_{\alpha \beta \gamma \delta}\left(x_{1}, x_{2} ; y_{1}, y_{2}\right)+ \\
& +\mathrm{i}\left[G_{\alpha \delta}\left(x_{1}, y_{2}\right) G_{\beta \gamma}\left(x_{2}, y_{1}\right)-G_{\alpha \gamma}\left(x_{1}, y_{1}\right) G_{\beta \delta}\left(x_{2}, y_{2}\right)\right] .
\end{aligned}
$$

To order $g^{0}$, the complete 4-point function contains only reducible parts,

$$
G_{\alpha \beta \gamma \delta}^{0}\left(x_{1}, x_{2} ; y_{1}, y_{2}\right)=G_{\alpha \delta}^{0}\left(x_{1}, y_{2}\right) G_{\beta \gamma}^{0}\left(x_{2}, y_{1}\right)-G_{\alpha \gamma}^{0}\left(x_{1}, y_{1}\right) G_{\beta \delta}^{0}\left(x_{2}, y_{2}\right) .
$$

This represents the Hartree-Fock part of the 4-point function. All remaining parts include interaction among the particles. Using $\bar{G}^{0}$ defined in Eq. (44), we find that $\bar{G}_{\alpha \beta \gamma \delta}^{0}\left(x_{1}, x_{2} ; y_{1}, y_{2}\right)=0$. The diagrams corresponding to Eq. (45) are shown in Fig. 6 .

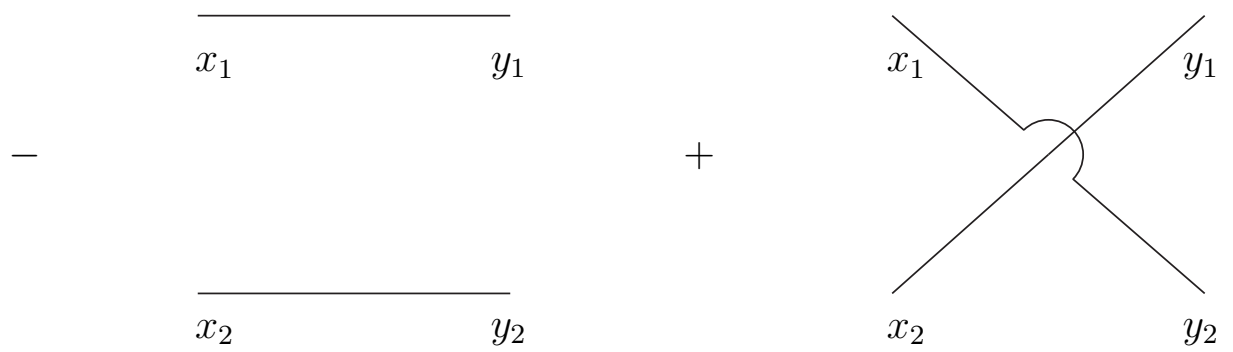

Figure 6: Diagrams for $g^{0}$-order of the 4-point function for the quartic potential

The irreducible parts appear in the first order in $g$,

$$
\begin{aligned}
\bar{G}_{\alpha \beta \gamma \delta}^{(1)}\left(x_{1}, x_{2} ; y_{1}, y_{2}\right) & =-2 g \int d z^{5}\left[G_{\alpha \bar{\alpha}}^{0}\left(x_{1}, z\right) G_{\bar{\alpha} \delta}^{0}\left(z, y_{2}\right) G_{\beta \bar{\beta}}^{0}\left(x_{2}, z\right) G_{\bar{\beta} \gamma}^{0}\left(z, y_{1}\right)\right. \\
& \left.-\left(\left(\delta, y_{2}\right) \leftrightarrow\left(\gamma, y_{1}\right)\right)\right],
\end{aligned}
$$

where $\left(\delta, y_{2}\right) \leftrightarrow\left(\gamma, y_{1}\right)$ means that there is an additional term in the square brackets, which can be obtained from the first one by replacing $\left(\delta, y_{2}\right)$ with $\left(\gamma, y_{1}\right)$ and vice versa. These parts are represented diagrammatically in Fig. 7. 


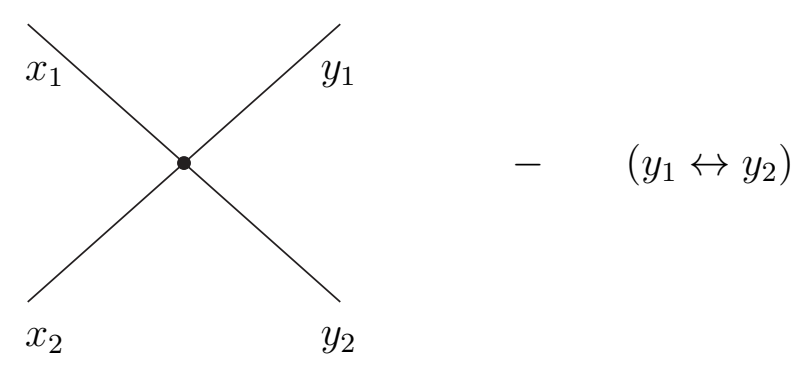

Figure 7: Diagrams for $g^{1}$-order of the irreducible 4-point Green's function for the quartic potential

The second order in $g$ contribution to the irreducible 4-point function is

$$
\begin{aligned}
& \bar{G}_{\alpha \beta \gamma \delta}^{(2)}\left(x_{1}, x_{2} ; y_{1}, y_{2}\right)=\mathrm{i}\left[G_{\beta \gamma}^{(1)}\left(x_{2}, y_{1}\right) G_{\alpha \delta}^{(1)}\left(x_{1}, y_{2}\right)-G_{\alpha \gamma}^{(1)}\left(x_{1}, y_{1}\right) G_{\beta \delta}^{(1)}\left(x_{2}, y_{2}\right)\right]+ \\
& +\left.\mathrm{i} \frac{g^{2}}{2} \int d^{5} z d^{5} w \frac{\delta^{4}}{\delta \bar{J}_{\alpha}\left(x_{1}\right) \delta \bar{J}_{\beta}\left(x_{2}\right) \delta J_{\gamma}\left(y_{1}\right) \delta J_{\delta}\left(y_{2}\right)}\left(T(z, w)-2 T_{0}(z) T(w)\right)\right|_{J=0=\bar{J}},
\end{aligned}
$$

that can be rewritten as

$$
\begin{aligned}
& \bar{G}_{\alpha \beta \gamma \delta}^{(2)}\left(x_{1}, x_{2} ; y_{1}, y_{2}\right)=-2 g \int d^{5} w\left[G_{\alpha \bar{\alpha}}^{0}\left(x_{1}, w\right) G_{\bar{\alpha} \delta}^{0}\left(w, y_{2}\right) G_{\beta \bar{\beta}}^{0}\left(x_{2}, w\right) G_{\bar{\beta} \gamma}^{(1)}\left(w, y_{1}\right)+\right. \\
& \quad+G_{\alpha \bar{\alpha}}^{0}\left(x_{1}, w\right) G_{\bar{\alpha} \delta}^{0}\left(w, y_{2}\right) G_{\beta \bar{\beta}}^{(1)}\left(x_{2}, w\right) G_{\bar{\beta} \gamma}^{0}\left(w, y_{1}\right)+ \\
& +G_{\alpha \bar{\alpha}}^{0}\left(x_{1}, w\right) G_{\bar{\alpha} \delta}^{(1)}\left(w, y_{2}\right) G_{\beta \bar{\beta}}^{0}\left(x_{2}, w\right) G_{\bar{\beta} \gamma}^{0}\left(w, y_{1}\right)+ \\
& \left.\quad+G_{\alpha \bar{\alpha}}^{(1)}\left(x_{1}, w\right) G_{\bar{\alpha} \delta}^{0}\left(w, y_{2}\right) G_{\beta \bar{\beta}}^{0}\left(x_{2}, w\right) G_{\bar{\beta} \gamma}^{0}\left(w, y_{1}\right)\right] \\
& -4 \mathrm{i} g^{2} \int d^{5} z d^{5} w\left[G_{\alpha \bar{\alpha}}^{0}\left(x_{1}, w\right) G_{\beta \bar{\beta}}^{0}\left(x_{2}, z\right) \Gamma_{\bar{\beta} \bar{\gamma}, \bar{\alpha} \bar{\delta}}(z, w) G_{\bar{\gamma} \gamma}^{0}\left(z, y_{1}\right) G_{\bar{\delta} \delta}^{0}\left(w, y_{2}\right)\right. \\
& \left.+G_{\alpha \bar{\alpha}}^{0}\left(x_{1}, w\right) G_{\bar{\alpha} \bar{\delta}}^{0}(w, z) G_{\bar{\delta} \delta}^{0}\left(z, y_{2}\right) G_{\beta \bar{\beta}}^{0}\left(x_{2}, w\right) G_{\bar{\beta} \bar{\gamma}}^{0}(w, z) G_{\bar{\gamma} \gamma}^{0}\left(z, y_{1}\right)\right] \\
& \quad-\left(\left(\delta, y_{2}\right) \leftrightarrow\left(\gamma, y_{1}\right)\right) .
\end{aligned}
$$

Some diagrams representing these processes are shown in Fig. 8. Part (a) corresponds to the third line of the previous equation; there are three more similar diagrams with self-energy loop $\Sigma_{1}(0)$ on one of the remaining three legs. Part (b) represents line 6, and part (c) corresponds to line 5 of the equation above.

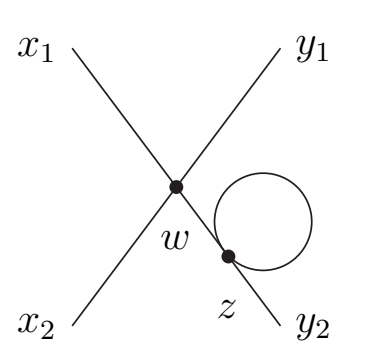

(a)

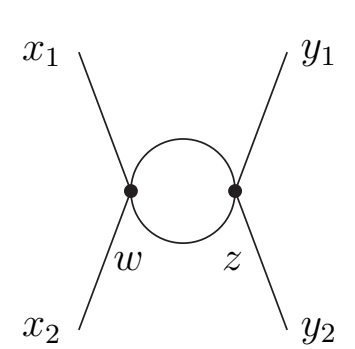

(b)

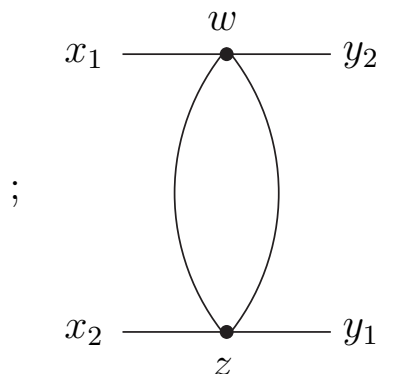

(c)

Figure 8: Diagrams for $g^{2}$-order of the irreducible 4-point Green's function for the quartic potential 
The function $\Gamma_{\bar{\beta} \bar{\gamma}, \bar{\alpha} \delta}(z, w)$ is defined as

$$
\begin{aligned}
\Gamma_{\bar{\beta} \bar{\gamma}, \bar{\alpha} \bar{\delta}}(z, w) & \equiv G_{\bar{\beta} \bar{\delta}}^{0}(z, w) G_{\bar{\alpha} \bar{\gamma}}^{0}(w, z)+\delta_{\bar{\alpha} \bar{\delta}} \Gamma_{\bar{\beta} \bar{\gamma} \bar{\gamma}}(z, w) \\
& +\delta_{\bar{\beta} \bar{\gamma}} \Gamma_{\bar{\alpha} \bar{\delta}}(w, z)-3 \delta_{\bar{\alpha} \bar{\delta}} \delta_{\bar{\beta} \bar{\gamma} \bar{\gamma}} G_{\gamma \delta}^{0}(z, w) G_{\delta \gamma}^{0}(w, z),
\end{aligned}
$$

being represented by a closed loop with two vertices and four external lines, as in Fig. 8 (c). This function has the following symmetry property

$$
\Gamma_{\bar{\beta} \bar{\gamma}, \bar{\alpha} \bar{\delta}}(z, w)=\Gamma_{\bar{\alpha} \bar{\delta}, \bar{\beta} \bar{\gamma}}(w, z) .
$$

Taking $\bar{\beta}=\bar{\gamma}$ and summing over $\bar{\beta}$, this gives us

$$
\Gamma_{\bar{\beta} \bar{\beta}, \bar{\alpha} \bar{\delta}}(z, w)=3 \Gamma_{\bar{\alpha} \bar{\delta}}(w, z)-8 \delta_{\bar{\alpha} \bar{\delta}} G_{\gamma \delta}^{0}(z, w) G_{\delta \gamma}^{0}(w, z) .
$$

In a similar way, we obtain

$$
\Gamma_{\bar{\beta} \bar{\gamma}, \bar{\alpha} \bar{\alpha}}(z, w)=3 \Gamma_{\bar{\beta} \bar{\gamma}}(z, w)-8 \delta_{\bar{\beta} \bar{\gamma}} G_{\gamma \delta}^{0}(z, w) G_{\delta \gamma}^{0}(w, z) .
$$

The polarisation part arise from the 4-point function and provides a sum of the loops to arbitrary order. The equivalence to the case of non-relativistic many-body systems interacting by two-particle interactions is obvious. Here we have parts that may be considered for particles and anti-particles.

\section{Concluding remarks}

This paper is the continuation of our previous works on quantization of Galileancovariant field theories: path-integral quantization of complex scalar fields in Ref. [5] and the canonical quantization of both scalar and fermi fields in Ref. [20]. The main purpose of this approach is to exploit relativistic tensorial techniques for applications to non-relativistic many-body systems. It is also interesting to compare Lorentzian and Galilean theories. An example of a rather unexpected similarity is that the nonzero spin is also predicted within a Galilean framework coherently defined [27, 22]. The presence of antiparticles is another example. However, there is no creation of particle and antiparticle pairs. It may be emphasized that the antisymmetrisation of the 4-point functions for fermions is also clearly respected. In addition to many familiar dissimilarities, some deserve to be emphasized, such as the existence of two Galilean formulations of electrodynamics [25, 26].

We have discussed the Dirac equation on a $(4+1)$ manifold and its reduction to the Lévy-Leblond equations [22], and the coexistence of positive- and negativeenergy/mass solutions [19]. While doing so, a representation of the Dirac matrices different from what is used earlier is presented, as well as the related spinors. After discussing the Galilean generating functional and Green's functions for particles and antiparticles, we compute the 2- and 4-point functions for the self-interacting quartic potential. 
From this study, we find that the following observations on the use of a $(4+1)$ dimensional Galilean space-time are in order. There exists a mass superselection rule, which prevents the creation of massive particles. This makes Yukawa coupling to massive particles irrelevant, because only couplings that involve at least four particles are allowed whereas Yukawa coupling to massless particles like the photon is possible.

An open question concerns the parity operator in Galilean field theories. The Clifford algebra theory asserts that there is no parity operator analogous to $\gamma^{5}$ in any odd-dimensions. We are currently investigating the possibility to embed the Galilean space-time into a $(5+1)$-dimensional Minkowski manifold, for which a parity operator exists with natural 8-dimensional Dirac gamma matrices [23]. This extention will allow us to study the Galilean analogue of the Nambu-Jona-Lasinio model [28].

In Poincaré covariant field theories in $(3+1)$ dimensions, $p_{\mu} p^{\mu}=E^{2}-\mathbf{p}^{2} c^{2}$ is an invariant and is equal to $m^{2} c^{4}$. This mass is an invariant quantity. However, in Galilean covariant field theories in $(4+1)$ dimensions, we have that $p_{\mu} p^{\mu}=2 m E-\mathbf{p}^{2}$ is an invariant that is set equal to a constant $k^{2}$. It is important to emphasize that $m$ appears as central charge in the Galilean algebra and this leads to the definition of the five-momentum as $(\mathbf{p}, E / \bar{c}, m \bar{c})$. Thus the renormalization process would affect the invariant $k$ in the Galilean covariant theory.

Finally, with the set up of the functional form for the path-integral approach, this would allow us to write down the transition amplitudes, hence the cross-sections, with the usual process of combining the square of the transition amplitude and the necessary phase space. The formulation as presented here has established contact with the usual perturbation theory for non-relativistic systems. However, it is important to emphasize that a covariant Galilean field theory is compatible with the idea of particles with energy $E$ and mass $+m$, and antiparticles with energy $-E$ and mass $-m$.

\section{Acknowledgments}

We acknowledge partial support by the Natural Sciences and Engineering Research Council of Canada.

\section{References}

[1] A.A. Abrikosov, L.P. Gorkov, I.E. Dzyaloshinski, Methods of Quantum Field Theory in Statistical Physics, Dover, New York 1963;

E.M. Lifshitz, L.P. Pitaevskii, Statistical Physics, Part 2 : Theory of the Condensed State, Landau and Lifshitz Course of Theoretical Physics, Vol. 9, Pergamon Press, Oxford, 1980;

A.M. Tsvelik, Quantum Field Theory in Condensed Matter Physics, Cambridge University Press, New York, 2003. 
[2] A. L. Fetter, J. D. Walecka, Quantum Theory of Many-Particle Systems, McGraw-Hill, New York, 1971.

[3] M. Greiner, C.A. Regal, D.S. Jin, Nature 426 (2003) 537.

[4] J. Lukierski, P.C. Stichel, W.J. Zakrzewski, Phys. Lett. A 357 (2006) 1;

M.A. del Olmo, M.S. Plyushchay, Ann. Phys. (NY) 321 (2006) 2830;

P.A. Horvathy, L. Martina, P.C. Stichel, Phys. Lett. B 564 (2003) 149;

P.A. Horvathy, M.S. Plyushchay, Nucl. Phys. B 714 (2005) 269;

P.A. Horvathy, L. Martina, P.C. Stichel, Phys. Lett. B 615 (2005) 87;

R. Jackiw, V.P. Nair, Phys. Lett. B 480 (2000) 237;

R. Jackiw, V.P. Nair, Phys. Lett. B 551 (2003) 166;

C. Duval, P.A. Horvathy, Phys. Lett. B 479 (2000) 284;

C. Duval, P.A. Horvathy, J. Phys. A: Math. Gen. 34 (2001) 10097;

C. Duval, P.A. Horvathy, Phys. Lett. B 547 (2002) 306.

[5] L. Abreu, M. de Montigny, F.C. Khanna, A.E. Santana, Ann. Phys. (N.Y.) 308 (2003) 244.

[6] M. de Montigny, F.C. Khanna, A.E. Santana, E.S. Santos, J.D.M. Vianna, Ann. Phys. (N.Y.) 277 (1999) 144.

[7] M. de Montigny, F.C. Khanna, A.E. Santana, E.S. Santos, J.D.M. Vianna, J. Phys. A : Math. Gen. 33 (2000) L273;

M. de Montigny, F.C. Khanna, A.E. Santana, E.S. Santos, J. Phys. A : Math. Gen. 34 (2001) 8901;

M. de Montigny, F.C. Khanna, A.E. Santana, Int. J. Theor. Phys. 42 (2003) 649.

[8] M. de Montigny, F.C. Khanna, A.E. Santana, J. Phys. A: Math. Gen. 36 (2003) 2009.

[9] Y. Takahashi, Fortschr. Phys. 36 (1988) 63;

Y. Takahashi, Fortschr. Phys. 36 (1988) 83.

[10] M. Omote, S. Kamefuchi, T. Takahashi, Y. Ohnuki, Fortschr. Phys. 37 (1989) 933.

[11] G. Pinski, J. Math. Phys. 9 (1968) 1927;

D.E. Soper, Classical Field Theory (1976) Wiley and Sons, New York, Sect. 7.3;

C. Duval, G. Burdet, H.P. Künzle, M. Perrin, Phys. Rev. D 31 (1985) 1841; 
C. Duval, G. W. Gibbons, P. Horváthy, Phys. Rev. D 43 (1991) 3907;

H.P. Künzle, C. Duval, in 'Semantical Aspects of Spacetime Theories' (U. Majer and H.J. Schmidt, Eds.) p. 113, BI-Wissenschaftsverlag, Mannheim, 1994;

C. Duval, P. Horváthy, L. Palla, Ann. Phys. (NY) 249 (1996) 265.

[12] E. Kapuścik, Nuov. Cim. A 58 (1980) 113;

E. Kapuścik, Acta Phys. Pol. B 12 (1981) 81;

E. Kapuścik, Acta Phys. Pol. B 17 (1986) 569.

[13] R. Jackiw, Lectures on Fluid Dynamics - A Particle Theorist's View of Supersymmetric, Non-Abelian, Noncommutative Fluid Mechanics and d-Branes, Springer, Berlin, 2002. Available at ArXiv : physics/0010042;

D. Bazeia, R. Jackiw, Ann. Phys. (N.Y.) 270 (1998) 246;

R. Jackiw, A.P. Polychronakos, Comm. Math. Phys. 207 (1999) 107;

R. Jackiw, A.P. Polychronakos (1999) Dynamical Poincaré symmetry realized by field-dependent diffeomorphisms Proc. Steklov Inst. Math. 226 193. Available at ArXiv : hep-th/9809123.

[14] M. Hassaïne, P.A. Horváthy, Ann. Phys. (N.Y.) 282 (2000) 218;

M. Hassaïne, P.A. Horváthy, Lett. Math. Phys. 57 (2001) 33.

[15] L. Susskind, Phys. Rev. 165 (1968) 1535;

J.B. Kogut, D.E. Soper, Phys. Rev. D 1 (1970) 2901;

J. Kogut, L. Susskind, Phys. Rep. 8 (1973) 75.

[16] S. Weinberg, Phys. Rev. 150 (1966) 1313.

[17] T. Banks, W. Fischler, S.H. Shenker, L. Susskind, Phys. Rev. D 55 (1997) 5112.

[18] B. Zwiebach, A First Course in String Theory, Cambridge University Press, New York, 2004.

[19] A. Horzela, E. Kapuścik, Electromagnetic Phenomena, 3 (2003) 63.

[20] E.S. Santos, M. de Montigny, F.C. Khanna, Ann. Phys. (NY) 320 (2005) 21.

[21] E.S. Santos, M. de Montigny, F.C. Khanna, A.E. Santana, J. Phys. A: Math. Gen. 37 (2004) 9771.

[22] J.M. Lévy-Leblond, Comm. Math. Phys. 6 (1967) 286.

[23] M. Kobayashi, M. de Montigny, F. Khanna, in preparation. 
[24] A. Das, Field Theory : A Path Integral Approach, World Scientific, Singapore, 1993;

M.E. Peskin, D.V. Schroeder, An Introduction to Quantum Field Theory, Perseus Books, Reading, 1995;

S. Weinberg, Quantum Field Theory Vol. 1, Cambridge Univ. Press, New York 1995.

[25] M. Le Bellac, J.M. Lévy-Leblond, Nuov. Cim. B 14 (1973) 217.

[26] F.S. Crawford, Am. J. Phys. 60 (1992) 109;

A. Horzela, E. Kapuścik, C.A. Uzes, Am. J. Phys. 61 (1993) 471;

F.S. Crawford, Am. J. Phys. 61 (1993) 472.

[27] J.M. Lévy-Leblond, in 'Group Theory and Applications' (E.M. Loebl, Ed), Vol. II, p. 221, Academic Press, New York, 1971.

[28] Y. Nambu, G. Jona-Lasinio, Phys. Rev. 122 (1961) 345;

Y. Nambu, G. Jona-Lasinio, Phys. Rev. 124 (1961) 246. 